\title{
Space vector transients of three-phase transformers
}

\author{
Diego Bellan, \\ Department of Electronics, Information and Bioengineering, Politecnico di Milano, \\ Piazza Leonardo da Vinci 32, 20133 Milan \\ Italy
}

Received: April 28, 2020. Revised: August 3, 2020. Accepted: October 1, 2020. Published: October 22, 2020.

\begin{abstract}
This paper is devoted to the transient analysis of three-phase transformers by means of the space vector tool. Space vector definition is based on the Clarke transformation, operating in the time domain. Thus, the space vector is better suited to transient analysis when compared with approximate approaches based on the phasor symmetrical-component transformation. Spacevector equivalent circuits are derived for three-phase transformers with Wye and Delta connections. A case study, consisting in the transient due to a capacitor bank insertion, shows that the proposed space-vector approach can clearly evidence the asymmetrical transient behavior of the phase variables in terms of different peak levels and oscillations amplitude.
\end{abstract}

Keywords-Analytical methods, Clarke transformation, space vector, three-phase transformers, transient analysis.

\section{INTRODUCTION}

$\mathrm{T}$ RANSIENT analysis is a key point in modern power systems where a huge number of electronic and electromechanical components are scattered along the networks and are continuously connected and disconnected. The dynamic circuit elements included in such components produce overcurrents/voltages when the topology of the network is sharply changed by the operation of switches. Prediction and evaluation of overcurrents/voltages is of paramount importance in order to prevent malfunctioning and damage of other components and subsystems [1]-[4].

Usually, transient analysis of power systems is performed by resorting to numerical approaches implemented in well-known software programs like EMTP (ElectroMagnetic Transients Program) [2]-[3]. A numerical simulation approach, however, as a general rule, does not allow theoretical insight and deep understanding of a phenomenon. Thus, the derivation of analytical methods for the transient analysis of three-phase systems is of great interest for electrical engineers. The conventional approach for analytical solution of transients in three-phase power systems is performed by resorting to singlephase equivalent circuits based on transformation methods like the symmetrical components [3]. This approach, however, can provide only approximate results, since the time-domain behavior of the three phase variables (i.e., phase voltages and currents) can be very different even if the system has a perfect phase symmetry. Therefore, new analytical methods should be derived in order to provide a complete time-domain description of a three-phase circuit under transient conditions.

In the literature, the main contributions oriented to the analytical modeling of transients in three-phase circuits are based on the Clarke transformation [4]-[7]. In fact, since the Clarke transformation operates in the time domain, it seems the most suited analytical tool for the given objective. In particular, the related concept of space vector is a powerful tool, able to provide three-phase information in compact and meaningful form. Moreover, in previous works it was shown that the conventional state-equation method can be extended to space vectors, with the further advantage of reducing the dynamical order of the network under analysis [7].

The above considerations concerning the need for threephase analytical models for a proper analysis of transients in a three-phase circuit hold for a specific component like the threephase transformer. The relevant technical literature is very rich in contributions concerning numerical modeling of three-phase transformers oriented to transient analysis (e.g., [8]-[17]), but as far as analytical approaches are considered, the conventional approach foresees the use of a simple single-phase equivalent of the transformer. As mentioned before, such approach cannot provide the complete time-domain behavior of a three-phase transformer transient since the three phase variables (i.e., the voltages and the currents of the three phases) in general show different time-domain behaviors (e.g., different peak values).

In this paper, the space-vector analytical model of a three- 
phase transformer is introduced. Using space vectors (based on the Clarke transformation) allow the analysis of a three-phase transformer as an actual three-phase component by avoiding the introduction of a single-phase equivalent. Thus, it will be shown that the space-vector approach provides the complete description of three-phase transients putting into evidence the different behavior of the three phase variables.

In particular, the general methodology based on the state equations for the space vectors [7] is recalled in Section II. The basic space-vector equivalent circuit of a three-phase transformer is derived in Section III by treating the transformer as six magnetically coupled circuits. In Section IV equivalent circuits are derived for Wye and Delta connections of transformer windings. A case study is analyzed in Section V to show the capability of space vectors to fully represent the main features of three-phase transients. Conclusions are drawn in Section VI.

\section{Space Vector Definition AND Properties}

A space vector is a complex-valued function of time, based on the Clarke transformation of a triplet of phase variables (e.g., voltages or currents) in a three-phase system [7]. The Clarke transformation is obtained from the more general Park transformation by assuming fixed axes. In its rational form, the Clarke transformation of time-domain phase currents is defined as:

$$
\begin{gathered}
\boldsymbol{i}_{\boldsymbol{T}}=\left[\begin{array}{l}
i_{\alpha} \\
i_{\beta} \\
i_{0}
\end{array}\right]=\boldsymbol{T}\left[\begin{array}{l}
i_{a} \\
i_{b} \\
i_{c}
\end{array}\right]= \\
=\sqrt{\frac{2}{3}}\left[\begin{array}{ccc}
1 & -1 / 2 & -1 / 2 \\
0 & \sqrt{3} / 2 & -\sqrt{3} / 2 \\
1 / \sqrt{2} & 1 / \sqrt{2} & 1 / \sqrt{2}
\end{array}\right]\left[\begin{array}{l}
i_{a} \\
i_{b} \\
i_{c}
\end{array}\right]
\end{gathered}
$$

A similar expression holds for a triplet of time-domain phase voltages. The rational form of the transformation results in the orthogonality of the transformation matrix $\boldsymbol{T}$, i.e., $\boldsymbol{T}^{-\mathbf{1}}=\boldsymbol{T}^{\boldsymbol{t}}$.

The transformed time-domain variables $\alpha$ and $\beta$ are combined to define the related space vector:

$$
\bar{i}(t)=i_{\alpha}(t)+j i_{\beta}(t)
$$

where $j=\sqrt{-1}$. The above definition of space vector is consistent with the well-known property of the transformation matrix $\boldsymbol{T}$ to diagonalize inductance matrices (and capacitance/resistance matrices, if needed) under the assumption of phase circuit symmetry (i.e., equal self and mutual parameters):

$$
\boldsymbol{L}_{\boldsymbol{T}}=\boldsymbol{T} \boldsymbol{L} \boldsymbol{T}^{-\mathbf{1}}=\boldsymbol{T}\left[\begin{array}{ccc}
L_{p} & L_{m} & L_{m} \\
L_{m} & L_{p} & L_{m} \\
L_{m} & L_{m} & L_{p}
\end{array}\right] \boldsymbol{T}^{-\mathbf{1}}=
$$

$$
=\left[\begin{array}{ccc}
L_{\alpha} & 0 & 0 \\
0 & L_{\beta} & 0 \\
0 & 0 & L_{0}
\end{array}\right]
$$

In fact, the diagonalized matrix $\boldsymbol{L}_{\boldsymbol{T}}$ is such that $L_{\alpha}=L_{\beta}=$ $L_{p}-L_{m}$. Since similar results can be obtained for all the threephase components of a system, the resulting uncoupled circuits $\alpha$ and $\beta$ can be treated as one circuit (same topology and parameters) where each pair of $\alpha$ and $\beta$ variables is replaced by the corresponding space vector.

The resulting space vector circuit is a conventional circuit where the circuit variables consist of space vectors instead of the usual real variables for voltages and currents. In particular, in [7] it was shown that the conventional state-equation approach can be used to solve a space-vector dynamic circuit in the time domain. To this aim, it is worth recalling that the state equation is written as:

$$
\frac{d}{d t} \overline{\boldsymbol{x}}=\boldsymbol{F} \overline{\boldsymbol{x}}+\boldsymbol{B} \overline{\boldsymbol{u}}
$$

where $\overline{\boldsymbol{x}}$ is the vector of the space vectors of the $N$ state variables (i.e., $N^{\text {th }}$ order dynamic circuit), $\overline{\boldsymbol{u}}$ is the vector of the space vectors of the sources, $\boldsymbol{F}$ is the state matrix, and $\boldsymbol{B}$ is the input matrix.

The steady-state solution corresponding to sources of the form $\overline{\boldsymbol{u}}=\boldsymbol{U} e^{ \pm j \omega t}$ (i.e., sinusoidal inputs) can be readily obtained as:

$$
\overline{\boldsymbol{x}}_{s}(t)=\boldsymbol{X}_{S} e^{ \pm j \omega t}=( \pm j \omega \mathbf{1}-\boldsymbol{F})^{-1} \boldsymbol{B} \boldsymbol{U} e^{ \pm j \omega t}
$$

Thus, the complete solution for the generic space-vector state variable can be written as:

$$
\bar{x}_{k}(t)=\sum_{n=1}^{N} C_{k n} e^{\lambda_{n} t}+\bar{x}_{s k}(t)
$$

where $\left\{\lambda_{n}\right\}_{n=1}^{N}$ is the set of the eigenvalues of the state matrix $\boldsymbol{F}$ (under the assumption of $N$ distinct eigenvalues), and the set of constants $\left\{C_{k n}\right\}_{n=1}^{N}$ can be obtained by imposing initial values $\overline{\boldsymbol{x}}_{0}=\overline{\boldsymbol{x}}(0)-\overline{\boldsymbol{x}}_{s}(0)$.

Finally, it is worth recalling that the phase variables $a, b, c$ can be readily recovered from the corresponding space vector through the equations (in case of $i_{0}=0$ ):

$$
\begin{gathered}
i_{a}(t)=\sqrt{\frac{2}{3}} \operatorname{Re}(\bar{l}(t)) \\
i_{b}(t)=\sqrt{\frac{2}{3}} \operatorname{Re}\left(\bar{\alpha}^{2} \bar{l}(t)\right) \\
i_{c}(t)=\sqrt{\frac{2}{3}} \operatorname{Re}(\bar{\alpha} \bar{l}(t))
\end{gathered}
$$

where $\bar{\alpha}=e^{j 2 \pi / 3}$. 


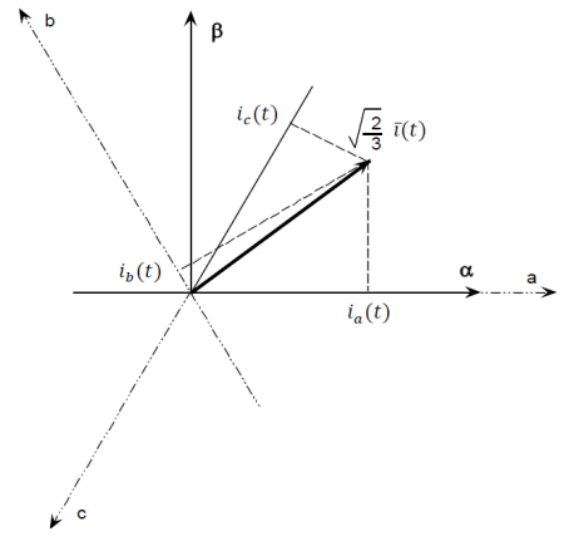

Fig. 1. The phase currents $i_{a}, i_{b}$, and $i_{c}$ can be recovered from the components of the space vector $\bar{l}$ on the axes $a, b$, and $c$.

Since the time behavior of the space vector is represented by a curve on the complex plane, from (7)-(9) we obtain that the instantaneous values of the phase variables $a, b, c$ can be recovered from the components of the instantaneous space vector on the three axes $a, b, c$, with geometrical phase displacement $2 \pi / 3$ each other, represented in Fig. 1.

In case of sinusoidal steady state, a space vector follows an ellipse shape on the complex plane with semi-major axis $R=\left|I_{p}\right|$ $+\left|I_{n}\right|$, semi-minor axis $r=\left|I_{p}\right|-\left|I_{n}\right| \mid$, and inclination angle $\varphi=$ $\left(\varphi_{p}-\varphi_{n}\right) / 2$, where $\varphi_{p}$ and $\varphi_{n}$ are the angles of the positivesequence phasor $I_{p}$ and the negative-sequence phasor $I_{n}$, respectively (according to the well-known symmetrical component transformation). If the negative-sequence current $I_{n}$ is equal to zero, the space vector follows a circular shape with radius $\left|I_{p}\right|$ on the complex plane.

\section{Space Vector Model of Coupled Three-Phase INDUCTORS}

In principle, a three-phase transformer consists in two threephase coupled inductors. Each three-phase inductor consists in three symmetrical inductors.

Therefore, we have a total of six magnetically-coupled circuits, grouped in two subsets of three circuits, i.e., the primary and the secondary three-phase windings. In Fig. 2 the terminals of the six circuits are put into evidence. Winding resistances $R_{1}$ and $R_{2}$ are also included in Fig. 2 .

The basic equations for the two three-phase inductors can be written as:

$$
\begin{aligned}
& {\left[\begin{array}{l}
v_{1 a} \\
v_{1 b} \\
v_{1 c}
\end{array}\right]=\left[\begin{array}{ccc}
R_{1} & 0 & 0 \\
0 & R_{1} & 0 \\
0 & 0 & R_{1}
\end{array}\right]\left[\begin{array}{l}
i_{1 a} \\
i_{1 b} \\
i_{1 c}
\end{array}\right]+\frac{d}{d t}\left[\begin{array}{l}
\psi_{1 a} \\
\psi_{1 b} \\
\psi_{1 c}
\end{array}\right]} \\
& {\left[\begin{array}{l}
v_{2 a} \\
v_{2 b} \\
v_{2 c}
\end{array}\right]=\left[\begin{array}{ccc}
R_{2} & 0 & 0 \\
0 & R_{2} & 0 \\
0 & 0 & R_{2}
\end{array}\right]\left[\begin{array}{l}
i_{2 a} \\
i_{2 b} \\
i_{2 c}
\end{array}\right]+\frac{d}{d t}\left[\begin{array}{l}
\psi_{2 a} \\
\psi_{2 b} \\
\psi_{2 c}
\end{array}\right]}
\end{aligned}
$$

where, in general, each flux is function of the six currents:

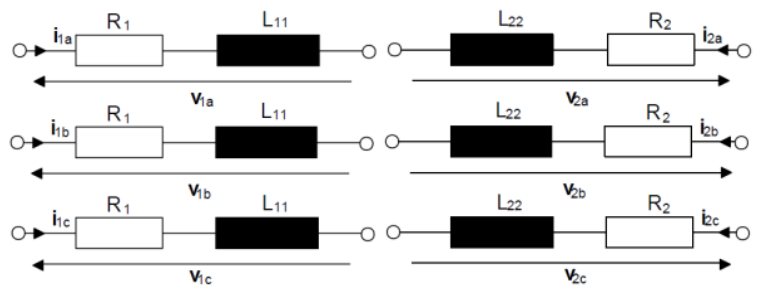

Fig. 2. The six magnetically-coupled circuits defining the primary and the secondary windings of a three-phase transformer.

$$
\left[\begin{array}{l}
\psi_{1 a} \\
\psi_{1 b} \\
\psi_{1 c} \\
\psi_{2 a} \\
\psi_{2 b} \\
\psi_{2 c}
\end{array}\right]=\left[\begin{array}{llllll}
L_{11} & M_{11} & M_{11} & L_{12} & M_{12} & M_{12} \\
M_{11} & L_{11} & M_{11} & M_{12} & L_{12} & M_{12} \\
M_{11} & M_{11} & L_{11} & M_{12} & M_{12} & L_{12} \\
L_{12} & M_{12} & M_{12} & L_{22} & M_{22} & M_{22} \\
M_{12} & L_{12} & M_{12} & M_{22} & L_{22} & M_{22} \\
M_{12} & M_{12} & L_{12} & M_{22} & M_{22} & L_{22}
\end{array}\right]\left[\begin{array}{c}
i_{1 a} \\
i_{1 b} \\
i_{1 c} \\
i_{2 a} \\
i_{2 b} \\
i_{2 c}
\end{array}\right]
$$

In a more compact matrix notation, (12) can be rewritten as:

$$
\left[\begin{array}{l}
\psi_{1} \\
\psi_{2}
\end{array}\right]=\left[\begin{array}{ll}
L_{1} & L_{M} \\
L_{M} & L_{2}
\end{array}\right]\left[\begin{array}{l}
i_{1} \\
i_{2}
\end{array}\right]
$$

Notice that the assumption of phase symmetry results in symmetrical coefficients in (12) and (13).

By substituting (13) into (10)-(11), and by using the Clarke transformation we obtain decoupled equations:

$$
\left[\begin{array}{l}
\boldsymbol{v}_{T 1} \\
\boldsymbol{v}_{T 2}
\end{array}\right]=\left[\begin{array}{cc}
\boldsymbol{R}_{1} & 0 \\
0 & \boldsymbol{R}_{2}
\end{array}\right]\left[\begin{array}{l}
\boldsymbol{i}_{T 1} \\
\boldsymbol{i}_{T 2}
\end{array}\right]+\left[\begin{array}{ll}
\boldsymbol{L}_{T 1} & \boldsymbol{M}_{\boldsymbol{T}} \\
\boldsymbol{M}_{\boldsymbol{T}} & \boldsymbol{L}_{T 2}
\end{array}\right] \frac{d}{d t}\left[\begin{array}{l}
\boldsymbol{i}_{T 1} \\
\boldsymbol{i}_{T 2}
\end{array}\right]
$$

where $\boldsymbol{v}_{T 1,2}$ and $\boldsymbol{i}_{T 1,2}$ are the $\alpha, \beta, 0$ vectors, $\boldsymbol{R}_{1,2}$ are diagonal resistance matrices, and:

$$
\begin{gathered}
\boldsymbol{L}_{T 1}=\left[\begin{array}{ccc}
L_{11}-M_{11} & 0 & 0 \\
0 & L_{11}-M_{11} & 0 \\
0 & 0 & L_{11}+2 M_{11}
\end{array}\right]= \\
\boldsymbol{L}_{T 2}=\left[\begin{array}{ccc}
L_{1} & 0 & 0 \\
0 & L_{1} & 0 \\
0 & 0 & L_{10}
\end{array}\right] \\
\boldsymbol{M}_{T}=\left[\begin{array}{ccc}
L_{22}-M_{22} & 0 & 0 \\
0 & L_{22}-M_{22} & 0 \\
0 & 0 & L_{22}+2 M_{22}
\end{array}\right]= \\
\left.=M_{12} \quad \begin{array}{ccc}
L_{2} & 0 & 0 \\
0 & L_{2} & 0 \\
0 & 0 & L_{20}
\end{array}\right] \\
\left.0 \quad \begin{array}{cc}
L_{12}-M_{12} \\
0 \\
0
\end{array}\right]= \\
=\left[\begin{array}{ccc}
L_{M} & 0 & 0 \\
0 & L_{M} & 0 \\
0 & 0 & L_{M 0}
\end{array}\right] .
\end{gathered}
$$


Thus, from (14)-(17) and by recalling the definition (2) of space vector, we obtain:

$$
\begin{aligned}
& \bar{v}_{1}=R_{1} \bar{i}_{1}+L_{1} \frac{d}{d t} \bar{i}_{1}+L_{M} \frac{d}{d t} \bar{i}_{2} \\
& \bar{v}_{2}=R_{2} \bar{i}_{2}+L_{M} \frac{d}{d t} \bar{i}_{1}+L_{2} \frac{d}{d t} \bar{i}_{2}
\end{aligned}
$$

Equations (18)-(19) represent the well-known equations of a two-port inductive network (i.e., two coupled inductors) with self-inductances $L_{1}$ and $L_{2}$, and mutual inductance $L_{M}$, where space vectors take the place of conventional currents and voltages. Therefore, according to basic circuit theory, several equivalent circuits can be derived for (18)-(19). A useful and widely used equivalent circuit based on an ideal transformer is shown in Fig. 3 where the transformer ratio is $k=L_{1} / L_{M}$, and the primary (parallel) and secondary (series) inductances are $L_{1}$ and $\sigma L_{2}$, respectively, with $\sigma=1-L_{M}^{2} /\left(L_{1} L_{2}\right)$.

The equivalent circuit in Fig. 3 holds in case the terminals of the six circuits in Fig. 2 correspond to the external terminals. In general, however, the terminals of the two three-phase inductors in Fig. 2 are Wye or Delta connected. Therefore, the terminals in Fig. 2 do not correspond to the external terminals of the transformer. The Wye and Delta connections, and the corresponding equivalent circuits at the external terminals are treated in Section IV.

\section{SPACE-Vector Equivalent Circuits For WyE AND DELTA CONNECTIONS}

Each of the two three-phase inductors in Fig. 2 can be Wye or Delta connected. In case of Wye connection, the star center can be accessible or not [18]. In the following Subsections the space-vector equivalent circuits of Wye and Delta connections are derived.

\section{A. Wye connection}

In the most general case, the star center of a Wye connection is accessible. The case of non-accessible star center can be regarded as a special case by setting zero star-center current.

Fig. 4 shows a Wye connection, and an arbitrary reference terminal $\mathrm{G}$ which allows proper definition of four ports, i.e., the three-phase port and the single-phase port connected to the star center. The four characteristic relationships of the Wye connection can be written as:

$$
\begin{gathered}
v_{a G}=v_{y G}, \quad v_{b G}=v_{y G}, \quad v_{c G}=v_{y G}, \\
i_{a}+i_{b}+i_{c}=i_{y} .
\end{gathered}
$$

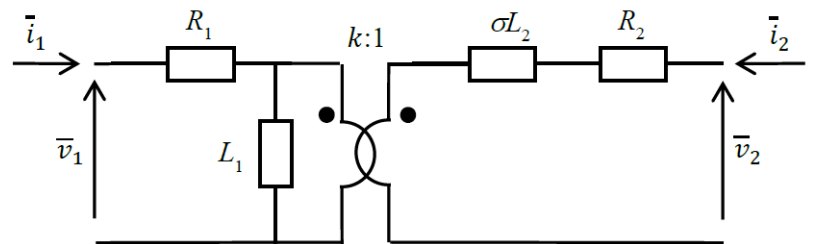

Fig. 3. Space-vector equivalent circuit of two three-phase symmetrical inductors.

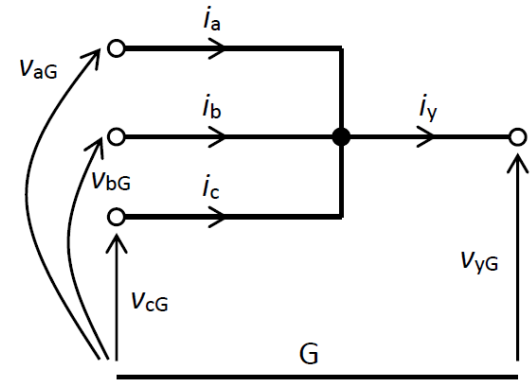

Fig. 4. Wye connection with accessible star center as a component with one three-phase port and one single-phase port.

The Clarke transformation of the phase variables $\left[\begin{array}{llll}v_{a G} & v_{b G} & v_{c G}\end{array}\right]^{T}$ provides $v_{\alpha}=v_{\beta}=0$. Therefore, the corresponding voltage space vector is $\bar{v}=0$. Thus, regardless the connection of the star center, in the space-vector domain a Wye connection is a short circuit to the reference terminal G. Indeed, connection of the star center impacts only on the zero component of the Clarke transformation, not investigated in this paper.

The series connection of a three-phase inductor and a Wye connection, therefore, has no impact on the space vectors. In fact, the space vector equivalent of the three-phase inductor is connected in series with a short circuit. Thus, if the primary side of the transformer is Wye connected, the corresponding spacevector equivalent is the primary side of the circuit in Fig. 3. If the secondary side of the transformer is Wye connected, the corresponding space-vector equivalent is the secondary side of the circuit in Fig. 3.

\section{B. Delta connection}

A Delta connection can be represented as in Fig. 5. External terminals are characterized by the voltages $\left[\begin{array}{lll}v_{a G} & v_{b G} & v_{c G}\end{array}\right]^{T}$ with respect to the reference terminal $\mathrm{G}$, whereas the inner terminals are characterized by the Delta voltages (i.e., the phase-to-phase voltages) $\left[\begin{array}{lll}v_{D a} & v_{D b} & v_{D c}\end{array}\right]^{T}$. The Delta voltages correspond to the voltages in Fig. 2 in case the three-phase inductor 1 or 2 is Delta connected.

The Delta connection in Fig. 5 is a component with six ports. Therefore, the required number of characteristic relationships is six, i.e., three relationships between the voltages and three relationships between the currents:

$$
\begin{aligned}
& {\left[\begin{array}{l}
v_{D a} \\
v_{D b} \\
v_{D c}
\end{array}\right]=\left[\begin{array}{ccc}
0 & 1 & -1 \\
-1 & 0 & 1 \\
1 & -1 & 0
\end{array}\right]\left[\begin{array}{l}
v_{a G} \\
v_{b G} \\
v_{c G}
\end{array}\right]=C\left[\begin{array}{l}
v_{a G} \\
v_{b G} \\
v_{c G}
\end{array}\right]} \\
& {\left[\begin{array}{l}
i_{a} \\
i_{b} \\
i_{c}
\end{array}\right]=\left[\begin{array}{ccc}
0 & -1 & 1 \\
1 & 0 & -1 \\
-1 & 1 & 0
\end{array}\right]\left[\begin{array}{l}
i_{D a} \\
i_{D b} \\
i_{D c}
\end{array}\right]=C^{T}\left[\begin{array}{l}
i_{D a} \\
i_{D b} \\
i_{D c}
\end{array}\right] .}
\end{aligned}
$$

By applying the Clarke transformation to (22)-(23) we obtain:

$$
\left[\begin{array}{l}
v_{D \alpha} \\
v_{D \beta} \\
v_{D 0}
\end{array}\right]=\boldsymbol{T} \boldsymbol{C} \boldsymbol{T}^{\boldsymbol{T}}\left[\begin{array}{l}
v_{\alpha} \\
v_{\beta} \\
v_{0}
\end{array}\right]
$$




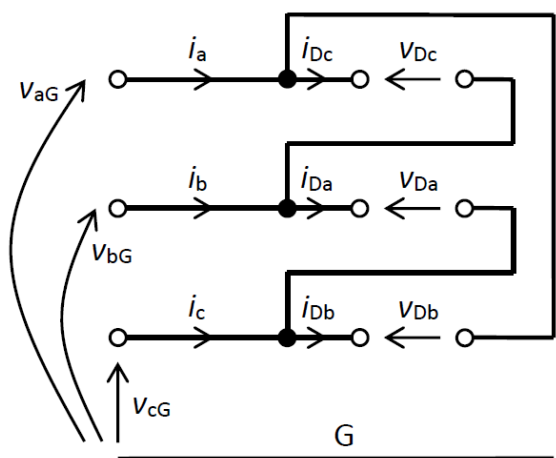

Fig. 5. Delta connection as a component with two three-phase ports.

$$
\left[\begin{array}{l}
i_{\alpha} \\
i_{\beta} \\
i_{0}
\end{array}\right]=\boldsymbol{T} \boldsymbol{C}^{\boldsymbol{T}} \boldsymbol{T}^{\boldsymbol{T}}\left[\begin{array}{l}
i_{D \alpha} \\
i_{D \beta} \\
i_{D 0}
\end{array}\right] .
$$

After simple algebra, from (24)-(25) we obtain the following relationships between the space vectors:

$$
\bar{v}=\frac{j}{\sqrt{3}} \bar{v}_{D}, \quad \bar{i}_{D}=\frac{-j}{\sqrt{3}} \bar{i}
$$

Therefore, in the space-vector domain, a Delta connection is represented by an ideal transformer with complex ratio

$$
\bar{k}_{D}=\frac{j}{\sqrt{3}}
$$

Thus, if the primary side of a transformer is Delta connected, then an ideal transformer with ratio (27) must be inserted on the primary side in Fig. 3. The resulting equivalent circuit is shown in Fig. 6a, whereas in Fig. 6b the elements $R_{1}$ and $L_{1}$ are reported on the left side of the ideal transformer (27), and the two cascaded ideal transformers are represented by one transformer with ratio $\bar{k}_{D} k$. Similarly, if the Delta connection is on the secondary side of the transformer, then an ideal transformer with ratio $\bar{k}_{D}^{-1}$ must be considered. The equivalent circuits are shown in Fig. 7.

\section{CASE STUdy}

In this Section the transient analysis of the three-phase system depicted in Fig. 8 including a Delta/Wye transformer was performed by using the results derived in the previous Sections. The correctness of all the proposed analytical results were checked through the Simulink implementation of the three-phase system.

The numerical values of the parameters in Fig. 8 were selected for illustration purpose only, i.e., they are not representative of a specific real system. The $50-\mathrm{Hz}$ three-phase voltage source was a balanced positive-sequence source with phase voltage $\bar{E}_{a}=1 \mathrm{kV} \mathrm{rms}$. The three-phase transformer was Delta-connected at primary side, and Wye-connected at secondary side. The transformer parameters were $k=10, R_{1}=$ $10 \Omega, R_{2}=1 \Omega, \sigma L_{2}=1 \mathrm{mH}$. The inductance $L_{1}$ was assumed much larger than $\sigma L_{2}$, such that it can be approximated as an

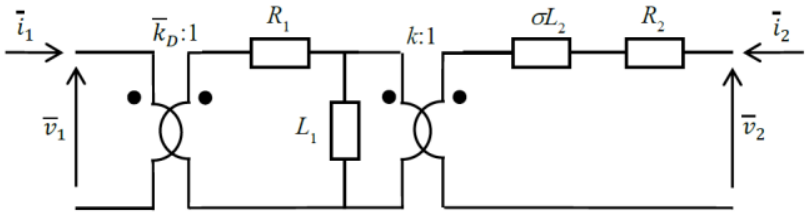

(a)

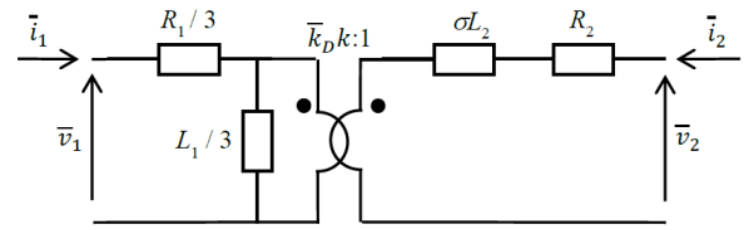

(b)

Fig. 6. Space-vector equivalent circuit of a three-phase transformer with Delta connection on the primary side (a). By reporting the elements $R_{1}$ and $L_{1}$ to the left side, one equivalent ideal transformer is obtained by cascading the two ideal transformers $\bar{k}_{D}$ and $k(\mathrm{~b})$.

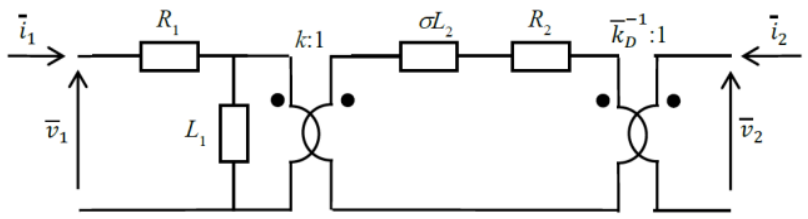

(a)

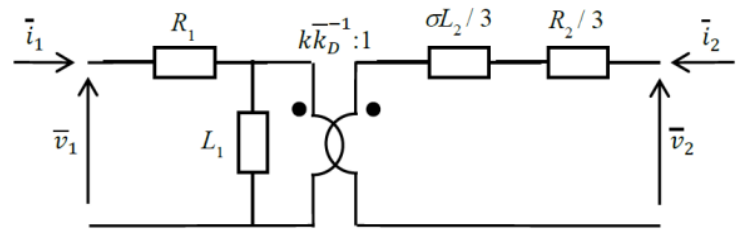

(b)

Fig. 7. Space-vector equivalent circuit of a three-phase transformer with Delta connection on the secondary side (a). By reporting the elements $\sigma L_{2}$ and $R_{2}$ to the right side, one equivalent ideal transformer is obtained by cascading the two ideal transformers $\bar{k}_{D}^{-1}$ and $k(\mathrm{~b})$.

open circuit. The load was $R=100 \Omega$ and $L=100 \mathrm{mH}$, whereas the capacitor bank inserted at $t=0$ was $C=50 \mu \mathrm{F}$.

According to Fig. 6, the space-vector equivalent circuit of the three-phase system in Fig. 8 is represented in Fig. 9. The timedomain solution of the circuit in Fig. 9 for $t \geq 0$ was obtained through the state equation (4) where:

$$
\begin{gathered}
\overline{\boldsymbol{x}}=\left[\begin{array}{c}
\bar{i} \\
\overline{i_{L}} \\
\bar{v}
\end{array}\right], \quad \bar{u}=\bar{e} \\
\boldsymbol{F}=\left[\begin{array}{ccc}
-\frac{R_{1}}{k^{2}+R_{2}} & 0 & -\frac{1}{\sigma L_{2}} \\
0 & -\frac{R}{L} & \frac{1}{L} \\
\frac{1}{C} & -\frac{1}{C} & 0
\end{array}\right], \quad \boldsymbol{B}=\left[\begin{array}{c}
1 \\
\bar{k}_{D^{k}} \\
0 \\
0
\end{array}\right]
\end{gathered}
$$




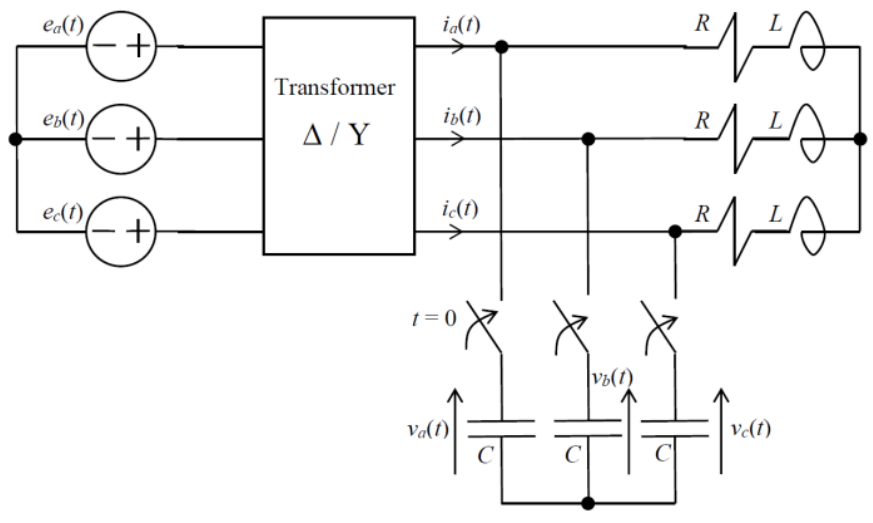

Fig. 8. The three-phase system used as a case study to illustrate the space-vector transient analysis.

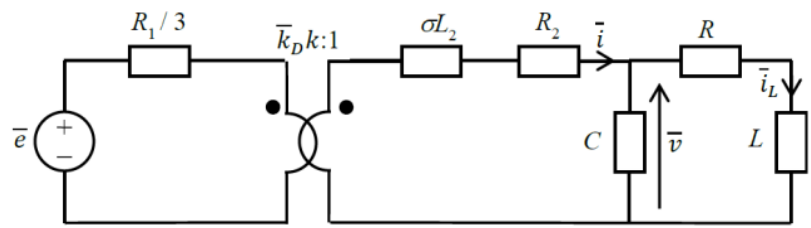

Fig. 9. Space-vector equivalent circuit of the three-phase system in Fig. 8.

Fig. 10 shows the trajectory of the space vector $\bar{i}(t)$ of the phase currents. The red curve shows the steady-state behavior for $t<0$. At $t=0$ the trajectory leaves the red curve (see the black arrow in the figure) and shows a transient behavior towards the new steady state. Notice that in steady state the space vector trajectory is a circle. In Fig. 10 the circles appear as ellipses because of the different scales of the real and imaginary parts, selected to improve the view of the transient. According to Fig. 1, the phase currents $i_{a, b, c}$ can be obtained as the components of the space vector on the axes $a, b, c$. The phase currents corresponding to Fig. 10 are represented in Fig. 11. Notice the large overcurrents $i_{b}$ and $i_{c}$ compared to $i_{a}$. Thus, despite the perfect symmetry of the three-phase system, the transient behavior of the phase currents is largely asymmetrical.

Fig. 12 shows the trajectory of the space vector $\bar{v}(t)$ of the voltages across the capacitors in Fig. 8. The transient is mainly in the vertical direction. Therefore, the phase $a$ (i.e., the real component of the space vector) is less involved in the transient. This is confirmed in Fig. 13, where the time behavior of the voltages across the capacitors are represented. Also in this case the asymmetry in the transient behavior of the three phase variables is clearly apparent.

Figs. 14-17 show the same variables as Figs. 10-13 with a smaller value of $R_{2}$ (i.e., $0.1 \Omega$ instead of $1 \Omega$ ). The damping of the oscillations in the transient behavior is clearly lower, i.e., currents and voltages have more persistent oscillating behavior and higher overcurrents/overvoltages can be observed.

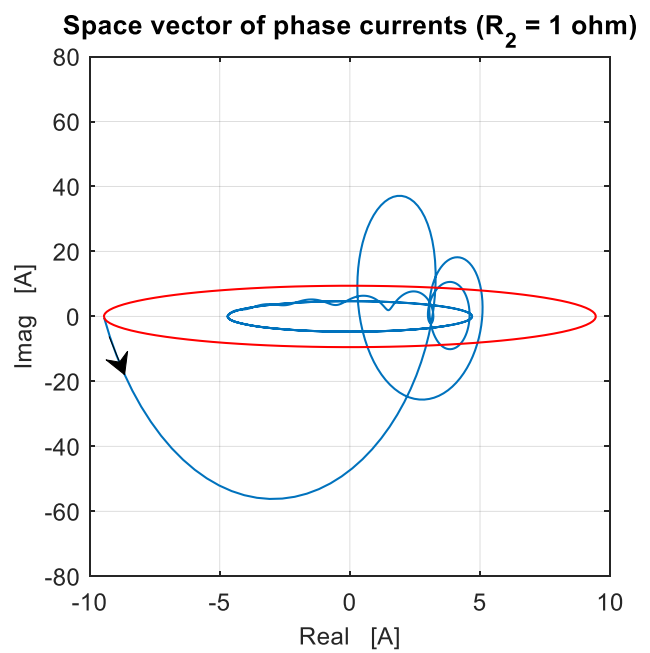

Fig. 10. Trajectory of the space vector $\bar{i}(t)$ of the phase currents in Fig. 8.

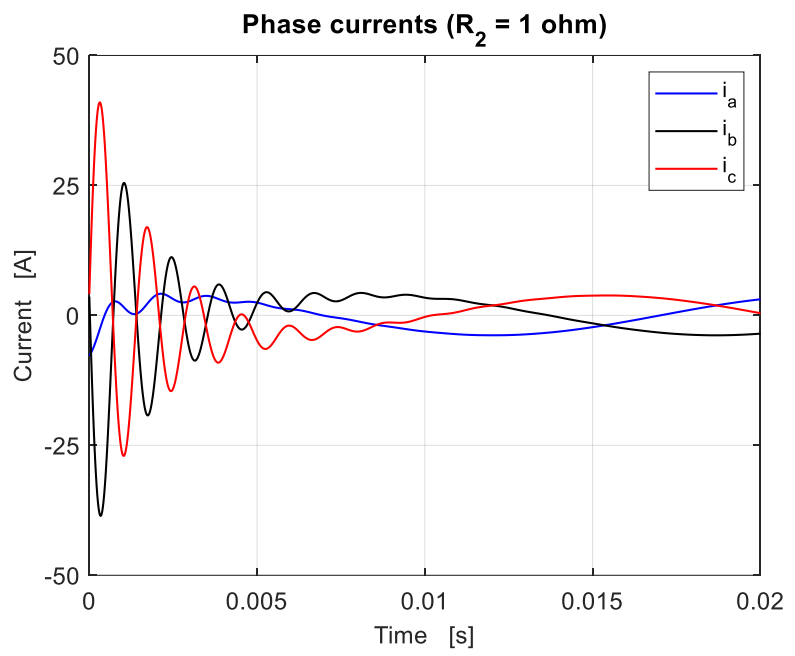

Fig. 11. Time-domain behavior of the phase currents $i_{a, b, c}$ in Fig. 8.

\section{CONCLUSION}

The space vector concept is intrinsically well-suited for the transient analysis of three-phase systems. The transient trajectory of a space vector on the complex plane has proven to be very rich in information about the time behavior of the three underlying phase variables. The extension of the state equation approach to space vectors allows the time-domain analysis of general dynamical circuits.

The space-vector equivalent circuit of three-phase transformers was introduced and clarified in the paper. The Wye and Delta connections were investigated, and the corresponding equivalent circuits were introduced through properly defined ideal transformers. The case study presented in the paper showed that even under the assumption of perfect symmetry of the three phases, the transient behavior of the phase variables is not symmetrical. This confirms that the space vector approach is better suited than any other kind of approach based on single-phase equivalents. 


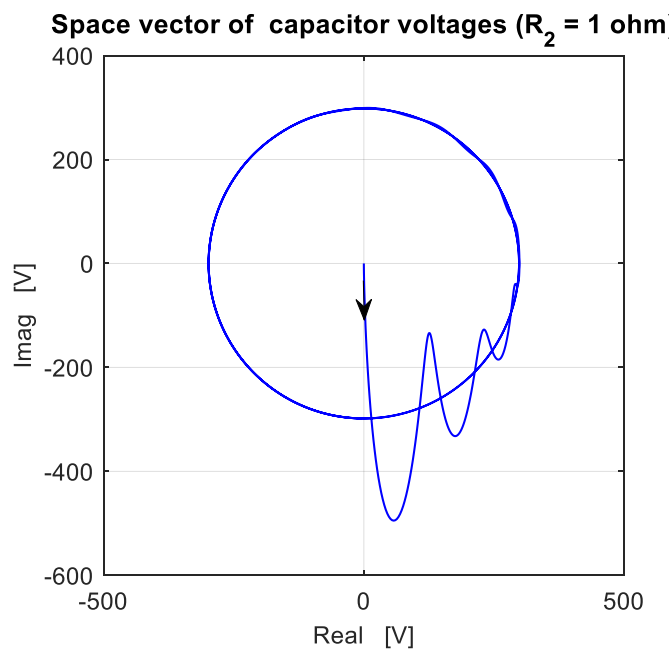

Fig. 12. Trajectory of the space vector $\bar{v}(t)$ of the voltages across the capacitors in Fig. 8.

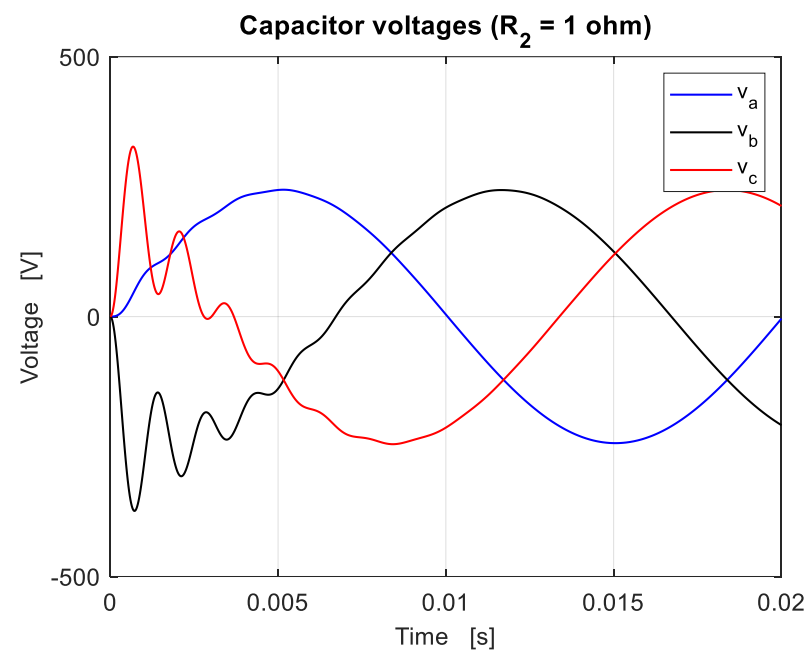

Fig. 13. Time-domain behavior of the capacitor voltages $v_{a, b, c}$ in Fig. 8.

\section{References}

[1] M. H. J. Bollen and I. Yu-Hua Gu, "On the analysis of voltage and current transients in three-phase power systems," IEEE Trans. on Power Delivery, vol. 22, pp. 1194-1201, 2007.

[2] J. Mahseredjian, V. Dinavahi, and J. A. Martinez, "Simulation tools for electromagnetic transients in power systems: overview and challenges," IEEE Trans. on Power Delivery, vol. 24, pp. 1657-1669, 2009.

[3] L. van der Sluis, Transients in power systems, John Wiley \& Sons Ltd, 2001.

[4] D. Bellan, "Clarke transformation solution of asymmetrical transients in three-phase circuits," Energies, vol. 13, pp. 1-19, 2020.

[5] M. Owen, "Transient analysis using component transforms," in Proc. of 2011 IEEE Power Engineering and Automation Conference, 2011.

[6] D. Bellan, "Analytical Approach to Transient Solution of Single-Line and Double-Line Faults in Three-Phase Circuits," International Journal of Circuits, Systems and Signal Processing, vol. 13, pp. 647-653, 2019.

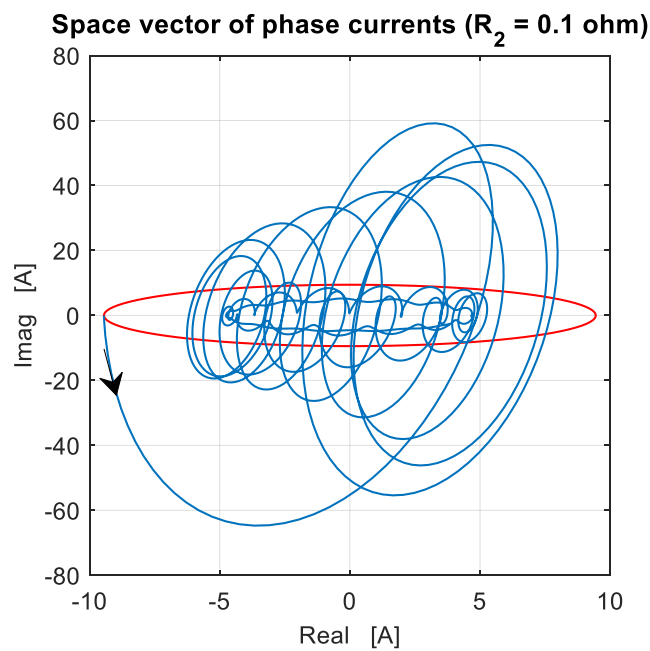

Fig. 14. Trajectory of the space vector $\bar{i}(t)$ of the phase currents in Fig. 8. Compared with Fig. 10, a smaller $R_{2}$ was selected.

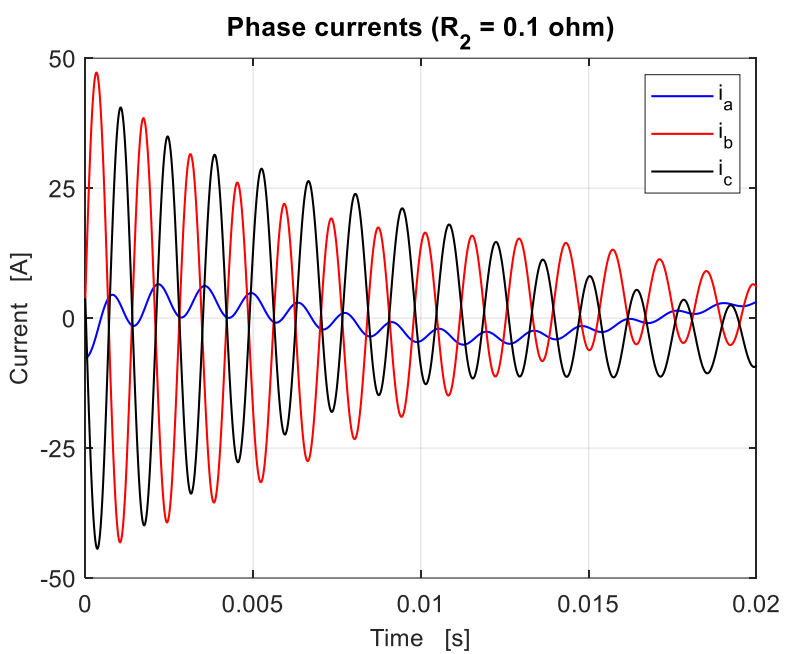

Fig. 15. Time-domain behavior of the phase currents $i_{a, b, c}$ in Fig. 8. Compared with Fig. 11, a smaller $R_{2}$ was selected.

[7] D. Bellan and G. Superti-Furga, "Space-vector stateequation analysis of three-phase transients," Journal of Electrical Systems, vol. 14, pp. 188-198, 2018.

[8] G. Bilal, J. M. Villanueva-Ramirez, and P. Gomez, "Frequency domain transformer model for electromagnetic transient analysis of networks," in Proc. of 2017 North American Power Symposium (NAPS), Morgantown, USA, 17-19 Sept., 2017.

[9] D. Tishuai, Z. Bide, F. Chunen, L. Wei, R. Xiao, and C. Chuanjiang, "Influence of system transients on the residual flux of three-phase transformers," in Proc. of $20174^{\text {th }}$ International Conference on Electric Power Equipment, Xi'an, China, 22-25 Oct., 2017.

[10]F. Aboura, S. A. Touhami, A. I. Zama, R. Tahmi, O. Touhami, and K. Boughrara, "Fast and very fast electromagnetic transients of three-phase three limb transformers: Theoretical approach and experiments," in Proc. of 2015 International Aegean Conference on Electrical Machines \& Power Electronics, Side, Turkey, 24 Sept., 2015. 


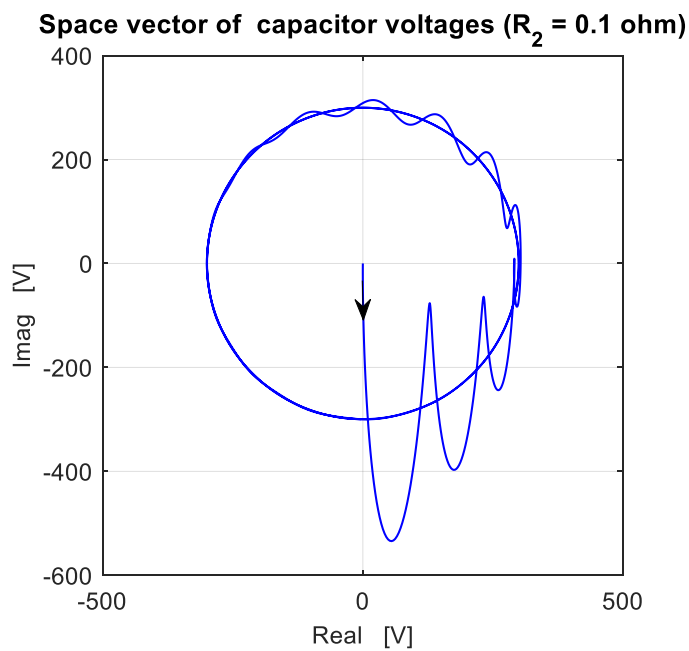

Fig. 16. Trajectory of the space vector $\bar{v}(t)$ of the voltages across the capacitors in Fig. 8. Compared with Fig. 12, a smaller $R_{2}$ was selected.

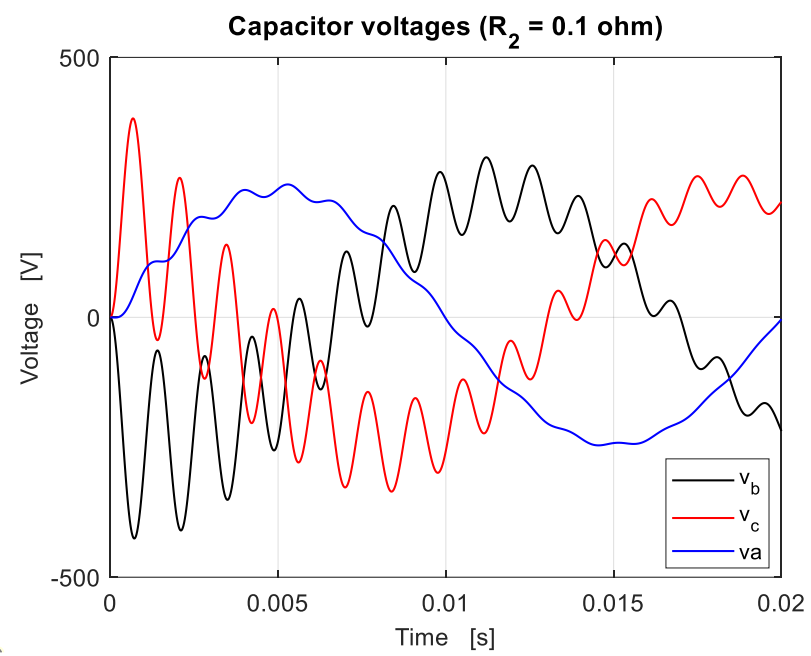

Fig. 17. Time-domain behavior of the capacitor voltages $v_{a, b, c}$ in Fig. 8. Compared with Fig. 13, a smaller $R_{2}$ was selected.
[11]M. Bolhasani, S. Kamangar, and S. Tavakoli, "Determination of distribution function of inrush current in three phase transformer using Monte Carlo method," in Proc. of 2012 47th International Universities Power Engineering Conference (UPEC), London, UK, 4-7 Sept., 2012.

[12]X. M. Lopez-Fernandez and C. Alvarez-Marino, "Computation Method for Transients in Power Transformers With Lossy Windings," IEEE Transactions on Magnetics, vol. 45, pp. 1863-1866, 2009.

[13] T. Abdulahović and T. Thiringer, "Voltage Stress in a Transformer Winding During Very Fast Transients Caused by Breaker Closing Event," IEEE Transactions on Power Delivery, vol. 29, pp. 1946-1954, 2014.

[14]P. Gómez, F. de León and F. P. Espino-Cortés, "Improved Computation of Core Inductance for Fast Transient Analysis of Transformers," IEEE Transactions on Power Delivery, vol. 29, pp. 2034-2036, 2014.

[15]B. A. Mork, F. Gonzalez, D. Ishchenko, D. L. Stuehm and J. Mitra, "Hybrid Transformer Model for Transient Simulation-Part I: Development and Parameters," IEEE Transactions on Power Delivery, vol. 22, pp. 248-255, 2007.

[16] M. Popov, L. van der Sluis, G. C. Paap and H. De Herdt, "Computation of very fast transient overvoltages in transformer windings," IEEE Transactions on Power Delivery, vol. 18, pp. 1268-1274, 2003.

[17]F. Li, Y. Liu, A. G. Tarditi and Z. Li, "Simulation of FastRise Transients in a Large-Power Transformer Winding," IEEE Transactions on Electromagnetic Compatibility, vol. 62, pp. 478-488, 2020.

[18]D. Bellan, S. A. Pignari, and G. Superti-Furga, "Consistent circuit technique for zero-sequence currents evaluation in interconnected single/three-phase power networks," Journal of Electrical Systems, vol. 12, pp. 230-238, 2016.

\section{Creative Commons Attribution License 4.0 (Attribution 4.0 International, CC BY 4.0)}

This article is published under the terms of the Creative Commons Attribution License 4.0 https://creativecommons.org/licenses/by/4.0/deed.en_US 